\title{
A Randomized Clinical Trial to Evaluate Labial Alveolar Bone Thickness and Apical Root Resorption between Two Types of Brackets Using Cone - Beam Computed Tomography
}

\author{
Shaza Hammad, Ahmed Fouda, Nader Giacaman \\ Department of Orthodontics, Faculty of Dentistry, Mansoura University, Mansoura, Egypt \\ Email for correspondence: shazamohammad@yahoo.com
}

\begin{abstract}
Context: There has always been a concern in determining the relationship between orthodontic tooth movement and the consequent biological costs to the periodontium and tooth root. To date, fewer studies have investigated the relationship between bracket type and labial alveolar bone thickness (LABT) of incisors in both mandible and maxilla, using cone beam computerized tomography (CBCT) data. This information will strengthen our understanding of labial movement and provide insights concerning the changes that occur. The present aims in studying the same. Objective: To assess LABT and apical root resorption (ARR) of incisors in patients undergoing the first phase of orthodontic treatment with two types of brackets. Materials and Methods: 21 Angle's Class I patients (anterior crowding: $3-5 \mathrm{~mm}$; mean age: 17.58 years \pm 2.38 years) were included in the study and randomly divided into two groups: Group I ( $n=10$, self-ligating brackets) and Group II ( $n=11$, conventional preadjusted brackets). LABT in two levels (L1, L2) and ARR were evaluated in 152 incisors by using CBCT scans. The CBCT scans were assessed by the same researcher, requested both at the beginning (T0) and 6 months after the initiation of orthodontic treatment (T1), with a level of sensitivity fixed at $25 \%$ by using Ondemand three-dimensional application Software (Version 1 , Cybermed, South Korea). The two groups were compared with Student $t$-test (parametric data) and Mann-Whitney test (non-parametric data) while paired groups were compared by paired $t$-test with $5 \%$ significance level. Results: On comparing the changes in ARR of lower incisors, the results showed statistically, but not clinically, significant change for the conventional group. However, no significant difference changes were observed between both groups regarding LABT at L1, LABT at L2 and ARR of upper incisors. Conclusions: There was tendency of incisors to suffer less resorption when using self-ligating brackets, but the results were inconclusive and clinically insignificant.
\end{abstract}

Key words: Apical root resorption, labial alveolar bone, self-ligating brackets

\section{INTRODUCTION}

There has always been a concern in determining the relationship between orthodontic tooth movement and the consequent biological costs

\section{Quick Response Code Article Info:}

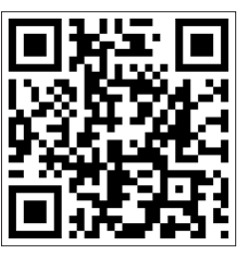

doi: 10.5866/2017.9.10210

Received: 04-12-2017

Revised: 23-12-2017

Accepted: 05-01-2018

Available Online: 15-02-2018, 2018 (www.

nacd.in)( NAD, 2018 - All rights reserved to the periodontium and tooth root. The initial stage of treatment crowding permanent teeth orthodontically is worried about teeth leveling and alignment. The accuracy of this procedure depends on various variables. Obviously, biological principles will perform an important role; the periodontium reaction towards the attached orthodontic force makes the main mechanism of permitting tooth movement inside the alveolar bone. ${ }^{[1]}$ Despite the importance of this biological element, it is somewhat behind the orthodontist hands, further concern should be attended with the option of bracket system. 
Frictional resistance created among the bracket slot and archwire has a considerable impact on tooth movement. Friction is generated upon the materialistic composition of different bracket and archwire types, and the technique of ligature between them. ${ }^{[2]}$ Several self-ligating brackets have been grown since 1935. Self-ligation doesn't need for an elastomeric attachment to catch the archwire, so it is related with a highly reduced friction with many different types of archwires, therefore less force required..$^{[3,4]}$ It is valuable to study any device system for its capability to move teeth, only with minimal injurious effects to the oral tissues.

The dense cortical plates are considered as orthodontic walls during orthodontic tooth repositioning. The loss of labial alveolar bone thickness (LABT) is a common complication with incisor moved labially. ${ }^{[5]}$ Nevertheless, it has been assumed that light orthodontic forces enhance better cellular activity than heavy forces in the surrounding tissues, which then not only improves the resorption process but also boosts bone apposition. ${ }^{[6,7]}$ Cone beam computerized tomography (CBCT) can be utilized to quantify bone thickness with high accuracy and reliability ${ }^{[8]}$

Apical root resorption (ARR) delivers with a multifactorial etiology. The severity of ARR has been shown to be positively related to the distance of tooth movement. ${ }^{[9]}$ Also, characteristics that are utilized for the orthodontic treatment, like type of brackets, mechanics applied, magnitude of forces and type of forces, are also relevant. ${ }^{[10]}$ Patients with noticeable root resorption through the first 6 months of active treatment are announced to be more eligible to root resorption after this period, and this is why radiographic examinations are advisable 6 months after corrective treatment, which influence orthodontist to modify or continue the treatment plan as necessary. ${ }^{[10,11]}$ CBCT scans have been glorified as the most accurate tool in identifying root resorption. ${ }^{[12]}$

Therefore, the aim of the present study was to guide practice by comparing the effects of passive self-ligating and conventional preadjusted brackets on LABT and ARR. To date, fewer studies have investigated the relationship between bracket type and LABT of incisors in both mandible and maxilla, using CBCT data. This information will strengthen our understanding of labial movement and provide insights concerning the changes that occur.

\section{MATERIALS AND METHODS}

Based on previous research, it was assumed that a sample size of 19 patients would give an $80 \%$ probability of detecting a real difference of $0.4 \mathrm{~mm}$ between groups at a statistically significant level of $5 \% .{ }^{[13]}$ Based on this assumption, the required sample size of this parallel-group study was 19 participants, and it was decided to round up to 21 subjects to make provisions for any losses. The patients were selected and participated in the study according to the followed criteria: Age ranged between 14 and 20 years, Angle's Class I malocclusion, crowding anteriorly ranging from 3 to $5 \mathrm{~mm}$, complete permanent dentition (excluding third molars). Patients with high-up canines, evidence of periodontal or gingival problems, signs of ARR or root canal treatment detected at the first examination or who went to previous orthodontic treatment were not participated. The patients received information about the future clinical trial and were signed on the informed consent sheet.

To eliminate the bias factor, a double blinded randomization was accomplished; the details of the series were unknown to any of the investigator or to the subjects. The patients were randomly allocated by sequentially numbered, opaque, sealed envelopes into two groups. The envelopes were opened only after writing the patient's tracking information on the envelope so that rendered as an audit trail.

The patients were divided into two groups: Group I were treated using passive self-ligating brackets (Clarity SL MBT brackets; 3M Unitek, Monrovia, CA, USA), and Group II: Were treated using conventional brackets ligated with elastomeric ligature (Gemini MBT brackets; 3M Unitek, Monrovia, CA, USA). For both groups, the patients were treated orthodontically within the initial leveling and alignment for the 6 months duration beginning with the same sequence of $0.014,0.016,0.016 \times 0.022$ inch nickel-titanium (NiTi) archwires and $0.016 \times 0.022$ inch stainlesssteel (SS) archwire. Each archwire was remained for 1.5 months, and it was replaced with the previously mentioned sequence. Dental stripping and extraction of premolars were eliminated.

CBCT were obtained from both groups in two time intervals, before beginning the orthodontic treatment and 6 months after it. All CBCT scans were carried out by a single experienced radiologist using the same tomographer (i-Cat Imaging Sciences 
International, Hatfield, $\mathrm{Pa}$ ). The specifications are the following: $13 \mathrm{~cm} \times 16 \mathrm{~cm}$ FOV, $8.9 \mathrm{~s}, 120 \mathrm{kVp}$, $5 \mathrm{~mA}$. This tomography has low-resolution sensors and affords $0.4 \mathrm{~mm}$ visual images. After scanning, all digital imaging and communications in medicine raw data are exported and saved. Then, it was imported into a specific CBCT software system (Ondemand three-dimensional [3D] App.). A specific tool inside the software (3D ceph analysis) is used to analyze the resultant images.

The CBCT scans were assessed by the same researcher in order to evaluate LABT in two levels (L1, L2) and ARR of upper and lower incisors with a level of sensitivity fixed at $25 \%$ by using Ondemand 3D App. Software (Version 1, Cybermed, South Korea).

\section{Image analysis}

Axial and sagittal cuts of upper and lower incisors in the center of the long axis were selected [Figure 1].

LABT of each incisor was determined at the site neighboring to the widest point of the labiopalatal root in two levels separated by $3 \mathrm{~mm}$ (L1 and L2, respectively); these levels were set along the long axis of the incisor and located every $3 \mathrm{~mm}$ from the cementoenamel junction level. LABT was assessed at the cervical level (L1) and midroot level (L2) at $\mathrm{T} 0$ and $\mathrm{T} 1$.

ARR was calculated by determining the difference in the total tooth length, which was measured in millimeters from the incisal edge to the root apex, between $\mathrm{T} 0$ and $\mathrm{T} 1$.

\section{Statistical analysis}

The 3D measurements of 9 randomly selected images were taken a month later by the same operator in order to determine intraexaminer errors by means of paired $t$-test (systematic errors) and Dahlberg formula (casual errors). ${ }^{[14]}$

Data were analyzed with SPSS Version 21. The normality of data was first tested with one-sample Kolmogorov-Smirnov test. Continuous variables were presented as mean \pm standard deviation for parametric data and median for non-parametric data. The two groups were compared with Student $t$-test (parametric data) and Mann-Whitney test (non-parametric data) while paired groups compared by paired $t$-test.

For all above mentioned statistical tests done, the threshold of significance is fixed at $5 \%$.

\section{RESULTS}

Total, 21 patients were enrolled; of these, 19 patients were received an intervention. Two patients from the Clarity SL group had not received the intervention for different reasons; however, the data from both groups were obtained and analyzed [Figure 2]. There was little difference between both groups in terms of demographic characteristics [Table 1].

Intraexaminer agreement was excellent $(P=0.721$ and Dahlberg $=0.23)$. Coefficients showed high rates of agreement for the measures with CBCT.

For Group I, no significant changes were observed in upper incisors regarding LABT at L1, L2 and ARR. Also, no significant changes were observed in lower incisors regarding LABT at L2 and ARR. However, a significant change was observed in lower incisors regarding LABT at L1 [Table 2].

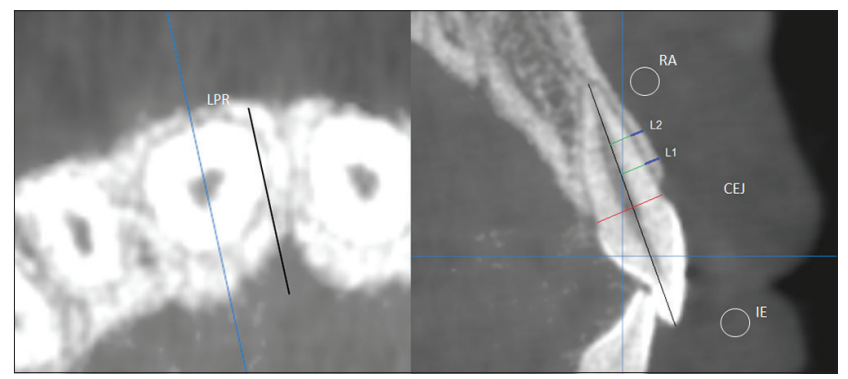

Figure 1: Determination of labial alveolar bone thickness and apical root resorption measurements

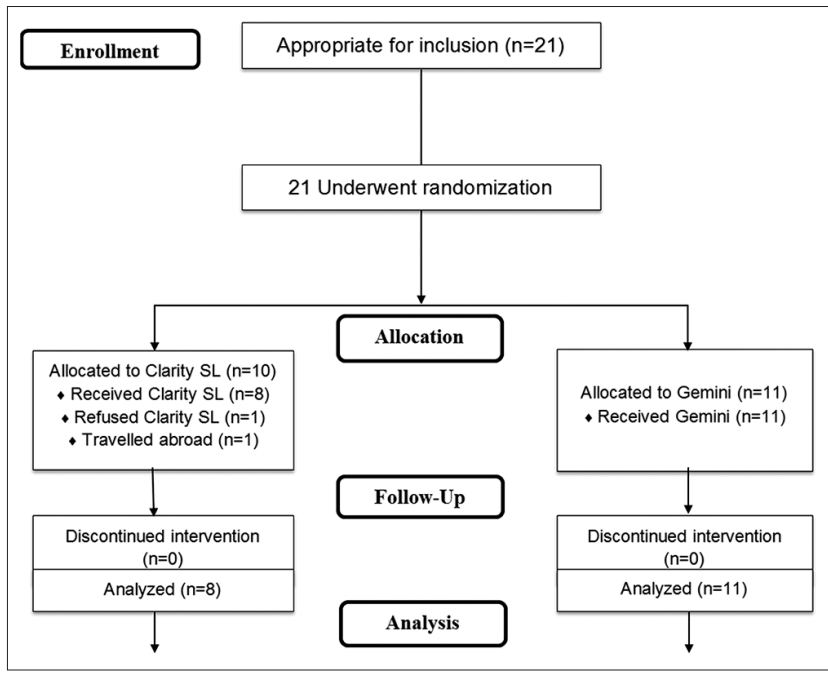

Figure 2: Consolidated standards of reporting trials flow diagram of participants during each stage of the trial 


\begin{tabular}{|c|c|c|c|c|c|}
\hline Items & $\begin{array}{l}\text { Total } \\
n=19(\%)\end{array}$ & $\begin{array}{c}\text { Group } 1 \\
n=8(\%)\end{array}$ & $\begin{array}{l}\text { Group } 2 \\
n=11(\%)\end{array}$ & Test of significant & $* \boldsymbol{P}$ \\
\hline \multicolumn{6}{|l|}{ Sex } \\
\hline Male & $6(31.6)$ & $2(25.0)$ & $4(36.4)$ & Fisher's exact & $1 \mathrm{NS}$ \\
\hline Female & $13(68.4)$ & $6(75.0)$ & $7(63.6)$ & & \\
\hline Age/years $($ Mean \pm SD $)$ & $17.58 \pm 2.38$ & $17.75 \pm 2.86$ & $17.45 \pm 2.11$ & $t=0.259$ & $\begin{array}{l}0.799 \\
\mathrm{NS}\end{array}$ \\
\hline \multicolumn{6}{|c|}{ Crowding (irregularity index, $\mathrm{mm}$ ) Mean $\pm \mathrm{SD}$} \\
\hline Upper arch & $3.76 \pm 1.46$ & $3.94 \pm 1.19$ & $3.64 \pm 1.68$ & $t=0.432$ & $\begin{array}{l}0.671 \\
\mathrm{NS}\end{array}$ \\
\hline Lower arch & $3.73 \pm 2.02$ & $3.56 \pm 2.05$ & $3.84 \pm 2.09$ & $t=0.293$ & $\begin{array}{l}0.773 \\
\mathrm{NS}\end{array}$ \\
\hline
\end{tabular}

*NS indicates no statistically significant difference. SD: Standard deviation

Table 2: Comparison of the degree of LABT at Level 1,

Level $2(\mathrm{~mm})$ and ARR ( $\mathrm{mm}$ ) between T0 and T1 for the participants in Group I (Clarity SL)

\begin{tabular}{lcccc} 
LABT & \multicolumn{2}{c}{ Group 1 } & Paired & $*$ \\
\cline { 2 - 3 } & Pre (T0) & Post $($ T1) & t-test & \\
\cline { 2 - 3 } & Mean \pm SD & Mean \pm SD & & \\
\cline { 1 - 2 } \multicolumn{2}{l}{ Upper incisors } & & & \\
L1 & $1.08 \pm 0.27$ & $1.00 \pm 0.29$ & 1.10 & 0.278 \\
& & & NS \\
L2 & $0.96 \pm 0.23$ & $1.06 \pm 0.33$ & 1.45 & 0.156 \\
& & & & NS \\
ARR & $22.53 \pm 1.69$ & $22.57 \pm 1.63$ & 0.25 & 0.797 \\
& & & & NS \\
Lower incisors & & & \\
L1 & $0.81 \pm 0.33$ & $0.57 \pm 0.22$ & 3.822 & $0.001^{*}$ \\
L2 & $0.68 \pm 0.34$ & $0.63 \pm 0.26$ & 1.353 & 0.186 \\
& & & & NS \\
ARR & $21.15 \pm 1.40$ & $21.30 \pm 1.33$ & 0.882 & 0.384 \\
& & & & $\mathrm{NS}$ \\
\hline
\end{tabular}

*Statistically significant difference $P<0.05$. NS indicates no statistically significant difference. LABT: Labial alveolar bone thickness, ARR: Apical root resorption, SD: Standard deviation

For Group II, no significant changes were observed in upper incisors regarding LABT at L1 and LABT at L2. Also, no significant change was detected in lower incisors regarding LABT at L2, while a significant change was observed in lower incisors regarding LABT at L1. Statistically significant changes were observed in upper and lower incisors regarding the ARR [Table 3].

On comparing the difference changes in upper and lower incisors in both groups regarding LABT at $\mathrm{L} 1$ and $\mathrm{LABT}$ at $\mathrm{L} 2$, the results revealed no
Table 3: Comparison of the degree of LABT at Level 1, Level $2(\mathrm{~mm})$ and ARR ( $\mathrm{mm}$ ) between T0 and T1 for the participants in Group II (Gemini)

\begin{tabular}{|c|c|c|c|c|}
\hline \multirow[t]{3}{*}{ LABT } & \multicolumn{2}{|c|}{ Group 2} & \multirow{3}{*}{$\begin{array}{c}\text { Paired } \\
t \text {-test }\end{array}$} & \multirow[t]{3}{*}{$* P$} \\
\hline & Pre (T0) & Post (T1) & & \\
\hline & Mean \pm SD & Mean \pm SD & & \\
\hline \multicolumn{5}{|c|}{ Upper incisors } \\
\hline L1 & $0.94 \pm 0.23$ & $0.84 \pm 0.30$ & 1.850 & $\begin{array}{c}0.071 \\
\mathrm{NS}\end{array}$ \\
\hline L2 & $1.08 \pm 0.38$ & $1.11 \pm 0.45$ & 0.499 & $\begin{array}{c}0.621 \\
\mathrm{NS}\end{array}$ \\
\hline ARR & $23.13 \pm 1.98$ & $22.93 \pm 2.00$ & 2.244 & $0.03 *$ \\
\hline \multicolumn{5}{|c|}{ Lower incisors } \\
\hline L1 & $0.72 \pm 0.26$ & $0.48 \pm 0.22$ & 5.075 & $<0.001^{*}$ \\
\hline L2 & $0.77 \pm 0.38$ & $0.69 \pm 0.31$ & 1.562 & $\begin{array}{c}0.126 \\
\mathrm{NS}\end{array}$ \\
\hline ARR & $22.21 \pm 1.526$ & $21.88 \pm 1.52$ & 2.972 & $0.005^{*}$ \\
\hline
\end{tabular}

*Statistically significant difference $P<0.05$. *NS indicates no statistically significant difference. LABT: Labial alveolar bone thickness, ARR: Apical root resorption, SD: Standard deviation

significant difference changes. On comparing the difference changes in upper incisors in both groups regarding ARR, the results revealed no significant difference changes. However, on comparing the difference changes in lower incisors in both groups regarding ARR, the results showed significant difference changes, which showed a statistically significant decrease in the ARR for Group II [Table 4].

\section{DISCUSSION}

In recent years, nonextraction treatment of crowded dentitions has become increasingly popular. New techniques and materials for expansion have 


\begin{tabular}{|c|c|c|c|c|c|c|c|c|}
\hline \multirow[t]{2}{*}{ Difference T1-T0 } & \multicolumn{3}{|c|}{ Group 1} & \multicolumn{3}{|c|}{ Group 2} & \multirow[t]{2}{*}{ Mann-Whitney test } & \multirow[t]{2}{*}{$* P$} \\
\hline & Mean & Median & SD & Mean & Median & SD & & \\
\hline \multicolumn{9}{|l|}{ Upper incisors } \\
\hline L1 & -0.07 & -0.06 & 0.38 & -0.09 & -0.03 & 0.35 & 0.262 & $\begin{array}{c}0.794 \\
\text { NS }\end{array}$ \\
\hline L2 & 0.10 & 0.06 & 0.40 & 0.02 & 0.04 & 0.32 & 0.940 & $\begin{array}{c}0.350 \\
\text { NS }\end{array}$ \\
\hline $\mathrm{ARR}$ & 0.03 & -0.03 & 0.72 & -0.20 & -0.02 & 0.59 & 1.546 & $\begin{array}{c}0.126 \\
\text { NS }\end{array}$ \\
\hline \multicolumn{9}{|l|}{ Lower incisors } \\
\hline L1 & -0.23 & -0.16 & 0.34 & -0.23 & -0.21 & 0.30 & 0.002 & $\begin{array}{c}0.998 \\
\text { NS }\end{array}$ \\
\hline L2 & -0.005 & -0.03 & 0.23 & -0.08 & -0.01 & 0.34 & 0.343 & $\begin{array}{c}0.733 \\
\text { NS }\end{array}$ \\
\hline ARR & 0.14 & 0.02 & 0.94 & -0.32 & -0.04 & 0.72 & 2.469 & $0.016 *$ \\
\hline
\end{tabular}

Mann-Whitney test was used. * Statistically significant difference $P \leq 0.05$. NS indicates no statistically significant difference. ARR: Apical root resorption, SD: Standard deviation

led to a marked reduction in the percentage of premolar extractions. The predominant theme of expansion is a loss of torque control. The loss of torque control leads to uncontrolled tipping in the buccal/labial direction. And tipping appears to have deleterious effects on the surrounding tissues.

In the present study, the incisors ( $n=152$ teeth) were examined, which are the most reabsorbed and moved teeth during orthodontic treatment. ${ }^{[15]}$

If incisors proceed forward to gain space, LABT will goes thinner. ${ }^{[5]}$ Thinner alveolar bone related to orthodontic movement, the alveolus could be more disposed to microfractures, leading to decrease in the height of the alveolar bone. ${ }^{[16]}$ In our groups, upper and lower LABT didn't exist the same; indeed, mostly it reduced especially in the lower incisors. However, alveolar bone loss was more obvious at the cervical region (L1) than the midroot region (L2). Probably due to alignment and leveling with small round wires create uncontrolled tipping movements to the incisors. Such movements generate forces that applied to the incisors and focused mainly at the alveolar crest [Tables 1 and 2].

Many CBCT factors influence the visibility of thin bony structures such as the cortical bone. ${ }^{[17]}$ Lower voxels result in the detection of thinner cortical bone and greater image resolution. These features make results more reliable, but patients are exposed to a greater amount of radiation. ${ }^{[17]}$ Our results showed that LABT changes are so negligible which can be referred to measurement error. Since we were dealing with very small regions of bone, it was impossible to precisely identify thickness changes of under $0.4 \mathrm{~mm}$. LABT changes were between 0.01 and $0.21 \mathrm{~mm}$ as per our results. These changes can't be measured precisely and are inside the borders of error.

The insignificance on comparing the difference changes regarding the LABT in both groups can't be utilized as a choice standard between them. It is upon the expert to use passive self-ligating brackets and considering its points of interest. For example, the ability to preserve LABT indicates that light forces allow orthodontists to move the incisors labially with less harm to the alveolar bone. ${ }^{[18]}$

In our research, self-ligating group had some increased in tooth length $(0.03-0.14 \mathrm{~mm})$; this could be related to the anatomical differences measurements. An in vitro CBCT precision study was performed, in order to evaluate root length. Measurement differences ranged from $0.07 \mathrm{~mm}$ to $0.26 \mathrm{~mm}$ and occurred because anatomic differences were not considered clinically significant. ${ }^{[19]}$

Maxillary anterior teeth were previously mentioned as owning the biggest amount of ARR, and others not. ${ }^{[10,11,13,15]}$ The results of our study demonstrated a tendency of these teeth to have the lowest ARR, with an average of $0.20 \mathrm{~mm}$. Our findings are less than those found by Leite et al. who reported that upper incisors had $0.35 \mathrm{~mm}$ of 
ARR. ${ }^{[13]}$ The average found in other studies with fixed orthodontic therapy, but using periapical radiographies, was $0.53 \mathrm{~mm}$ and $0.76 \mathrm{~mm} .{ }^{[10,20]}$ This difference may occur due to the method used, since the time of treatment was similar, 22-26 weeks, as compared to 24 weeks in this study. Yet, for root analysis most studies used periapical radiographies with parallelism technique, although some teeth may have their image lengthened, suggesting amplification and processing errors. ${ }^{[12]}$ Incisor angulation may change accompanied by alignment and leveling, thus might influence tooth height in the $\mathrm{X}$-ray. ${ }^{[11]}$ Consequently, technological advancements made it credible to measure the ARR in a $3 \mathrm{D}$ dimensions, because of its accuracy in evaluating root resorption. ${ }^{[12]}$ An additional factor that could illustrate the smaller resorption in our study would be the use of NiTi archwires, which provide less force than SS archwires, hence influencing the degree of the ARR.

Concerning the magnitude of ARR in all incisors, an average of $0.35 \mathrm{~mm}$ was detected-a value near to that in the literature of $0.25 \mathrm{~mm}$ in the leveling and alignment phase. This amount of ARR is small and clinically insignificance. ${ }^{[21]}$

Comparing the degree of ARR between patients treated with different types of brackets was based on delivering light forces attributed to self-ligating brackets. The results in our study showed a statistically significant difference change in the ARR for Group II [Table 4]. Probably due to less frictional force ${ }^{[4]}$ In a study evaluating the $3 \mathrm{D}$ orthodontic force system suggested that adding elastic ligation increased the forces in the 3 planes. ${ }^{[22]}$ Clarity SL brackets when combined with NiTi archwires, can display lower frictional force resistance, perhaps belongs to the touch between clarity clips and archwires of the same alloy (NiTi). ${ }^{[23]}$ In relevant studies concluded that there was no difference in the degree of ARR when comparing self-ligating and conventional brackets after alignment and leveling. ${ }^{[13,24]}$

Based on the results of our research, passive selfligating brackets were able to stimulate less ARR than conventional preadjusted brackets measured by CBCT, regarding lower incisors. However, further long-term clinical studies are necessary to confirm the results observed in this research.

Finally, because the effect sizes of some changes were so small, our 19 subjects were insufficient to rule out the possibility of Type II errors. This would have established a statistically, but not clinically, significant change.

\section{CONCLUSION}

- On comparing the changes in upper and lower incisors in both groups regarding LABT at $\mathrm{L} 1$ and LABT at L2, the results revealed no significant difference changes.

- On comparing the changes in upper incisors in both groups regarding ARR, the results revealed no significant difference changes.

- On comparing the changes in lower incisors in both groups regarding ARR, the results showed a significant difference change, which showed a statistically significant decrease in the ARR for the conventional group.

\section{REFERENCES}

1. Sandy JR, Farndale RW, Meikle MC. Recent advances in understanding mechanically induced bone remodeling and their relevance to orthodontic theory and practice. Am J Orthod Dentofac Orthop 1993;103:212-22.

2. Ireland A, Sherriff M, McDonald F. Effect of bracket and wire composition on frictional forces. Eur J Orthod 1991;13:322-8.

3. Khambay B, Millett D, McHugh S. Evaluation of methods of archwire ligation on frictional resistance. Eur J Orthod 2004;26:327-32.

4. Berger JL. The influence of the SPEED bracket's selfligating design on force levels in tooth movement: A comparative in vitro study. Am J Orthod Dentofac Orthop 1990;97:219-28.

5. Garlock DT, Buschang PH, Araujo EA, Behrents RG, Kim KB. Evaluation of marginal alveolar bone in the anterior mandible with pretreatment and posttreatment computed tomography in nonextraction patients. Am J Orthod Dentofac Orthop 2016;149:192-201.

6. Tomizuka R, Shimizu Y, Kanetaka H, Suzuki A, Urayama S, Kikuchi M, et al. Histological evaluation of the effects of initially light and gradually increasing force on orthodontic tooth movement. Angle Orthod 2007;77:410-6.

7. Hibino K, Wong RW. Orthodontic tooth movement enhancing bony apposition in alveolar bony defect: A case report. Cases J 2009;2:116.

8. Timock AM, Cook V, McDonald T, Leo MC, Crowe J, Benninger BL, et al. Accuracy and reliability of buccal bone height and thickness measurements from cone-beam computed tomography imaging. Am J Orthod Dentofacial Orthop 2011;140:734-44.

9. Weltman B, Vig KW, Fields HW, Shanker S, Kaizar EE. Root resorption associated with orthodontic tooth movement: A systematic review. Am J Orthod Dentofac Orthop 2010;137:462-76.

10. Årtun J, Smale I, Behbehani F, Doppel D, Van’t Hof M, 
Kuijpers-Jagtman AM. Apical root resorption six and 12 months after initiation of fixed orthodontic appliance therapy. Angle Orthod 2005;75:919-26.

11. Apajalahti S, Peltola JS. Apical root resorption after orthodontic treatment-a retrospective study. Eur J Orthod 2007;29:408-12.

12. Sherrard JF, Rossouw PE, Benson BW, Carrillo R, Buschang PH. Accuracy and reliability of tooth and root lengths measured on cone-beam computed tomographs. Am J Orthod Dentofac Orthop 2010;137:100-8.

13. Leite V, Conti AC, Navarro R, Almeida M, OltramariNavarro P, Almeida R. Comparison of root resorption between self-ligating and conventional preadjusted brackets using cone beam computed tomography. Angle Orthod 2012;82:1078-82.

14. Dahlberg G. Statistical Methods for Medical and Biological Students. London, UK: George Allen and Unwin; 1940.

15. Janson GR, de Luca Canto G, Martins DR, Henriques JF, de Freitas MR. A radiographic comparison of apical root resorption after orthodontic treatment with 3 different fixed appliance techniques. Am J Orthod Dentofac Orthop 2000;118:262-73.

16. Verna C, Dalstra M, Lee TC, Cattaneo PM, Melsen B. Microcracks in the alveolar bone following orthodontic tooth movement: A morphological and morphometric study. Eur J Orthod 2004;26:459-67.

17. Nowzari H, Molayem S, Chiu CH, Rich SK. Cone beam computed tomographic measurement of maxillary central incisors to determine prevalence of facial alveolar bone width 2 mm. Clin Implant Dent Relat Res 2012;14:595-602.

18. Thongudomporn U, Charoemratrote C, Jearapongpakorn S. Changes of anterior maxillary alveolar bone thickness following incisor proclination and extrusion. Angle Orthod 2014;85:549-54.

19. Lund H, Grondahl K, Gröndahl HG. Cone beam computed tomography for assessment of root length and marginal bone level during orthodontic treatment. Angle Orthod 2010;80:466-73.

20. Alexander SA. Levels of root resorption associated with continuous arch and sectional arch mechanics. Am J Orthod Dentofac Orthop 1996;110:321-4.

21. Malmgren O, Goldson L, Hill C, Orwin A, Petrini L, Lundberg M. Root resorption after orthodontic treatment of traumatized teeth. Am J Orthod 1982;82:487-91.

22. Badawi HM, Toogood RW, Carey JP, Heo G, Major PW. Three-dimensional orthodontic force measurements. Am J Orthod Dentofac Orthop 2009;136:518-28.

23. Monteiro MR, Silva LE, Elias CN, Od V. Frictional resistance of self-ligating versus conventional brackets in different bracket-archwire-angle combinations. J Appl Oral Sci 2014;22:228-34.

24. Handem RH, Janson G, Matias M, de Freitas KM, de Lima DV, Garib DG, et al. External root resorption with the self-ligating damon system-a retrospective study. Prog Orthod 2016;17:20. 